\title{
FAST NEUTRON BEAMS - PROSPECTS FOR THE COMING DECADE
}

\author{
Jan Blomgren* \\ Department of Neutron Research \\ Uppsala University \\ Box 525, S-751 20 Uppsala, Sweden \\ E-mail: Jan.Blomgren@tsl.uu.se
}

\begin{abstract}
The present status of neutron beam facilities above $20 \mathrm{MeV}$ is reviewed. Presently, two main techniques for neutron production at these energies are used; white beams and quasi-monoenergetic beams. The performances of these two techniques are discussed, as well as the use of such facilities for measurements of nuclear data for fundamental and applied research. Recently, two novel ideas on how to produce extremely intense neuton beams in the $100-500 \mathrm{MeV}$ range have been proposed. Decay in flight of beta-delayed neutron-emitting nuclei could provide beam intensities five orders of magnitudes larger than present facilities. A typical neutron energy spectrum would be essentially mono-energetic, i.e., the energy spread is about $1 \mathrm{MeV}$ with essentially no lowenergy tail. A second option would be to produce beams of ${ }^{6} \mathrm{He}$ and dissociate the ${ }^{6} \mathrm{He}$ nuclei into $\alpha$ particles and neutrons. The basic features of these concept are outlined, and the potential for improved nuclear data research is discussed.
\end{abstract}

International Workshop on Fast Neutron Detectors and Applications

April, 3 - 6, 2006

University of Cape Town, South Africa

${ }^{*}$ Speaker. 


\section{Introduction}

The interest in high-energy neutron data is rapidly growing since a number of potential largescale applications involving fast neutrons are under development, or have been identified. This has motivated nuclear data research for transmutation of spent nuclear fuel $[1,2,3]$, neutron therapy of cancer tumours [4] and upsets in electronics [5, 6]. In the present paper, present and future facilities for nuclear data production for these applications are discussed.

\section{Present-day facilities for nuclear data measurements}

At low energies (below $20 \mathrm{MeV}$ or so), truly mono-energetic neutron beams can be produced. There are a few light-ion reactions, like $\mathrm{D}(\mathrm{d}, \mathrm{n})^{3} \mathrm{He}$ and $\mathrm{T}(\mathrm{d}, \mathrm{n})^{4} \mathrm{He}$, which have positive Q-values and sizeable cross sections. Such a beam is strictly monoenergetic up to about $2 \mathrm{MeV}$ incident deuteron energy. Above this energy, there is a possibility that the deuteron breaks up into a proton and a neutron. In reality, this is not a major obstacle until you get up to about $30 \mathrm{MeV}$ neutron energy, because the $\mathrm{T}(\mathrm{d}, \mathrm{n})^{4} \mathrm{He}$ cross section is so large that the breakup neutrons form only a small low-energy tail. At even higher energies though, the $\mathrm{T}(\mathrm{d}, \mathrm{n})^{4} \mathrm{He}$ cross section is smaller, making the total yield too low for most measurements.

The largest neutron separation energy is about $20 \mathrm{MeV}$, making truly monoenergetic beams impossible to produce above that energy. What is available at higher energies are quasi-monoenergetic beams, i.e., beams where a single energy dominates, but always accompanied by a low-energy tail.

At energies of $50 \mathrm{MeV}$ and up, three production reactions give reasonably monoenergetic beams. These are $\mathrm{D}(\mathrm{p}, \mathrm{n}),{ }^{6} \mathrm{Li}(\mathrm{p}, \mathrm{n})$ and ${ }^{7} \mathrm{Li}(\mathrm{p}, \mathrm{n})$. The first has a large cross section, but the drawback that the energy resolution of the full-energy neutrons cannot be better than $3 \mathrm{MeV}$ due to the Fermi motion of the neutron inside the deuteron. If a sharper energy definition is required, one of the two reactions using lithium is selected. They are about equally good, but there is a major practical difference: ${ }^{6} \mathrm{Li}$ is used in hydrogen bombs and is therefore not easily obtained, while ${ }^{7} \mathrm{Li}$ is provided at low cost. As one could expect, ${ }^{7} \mathrm{Li}(\mathrm{p}, \mathrm{n})$ is the most common production reaction for monoenergetic neutron beams. At $100 \mathrm{MeV}$, about $50 \%$ of the neutrons fall within $1 \mathrm{MeV}$ at maximum energy, while the remaining half are distributed about equally from maximum energy down to zero. This is the closest to monoenergetic conditions nature provides.

There is also a completely different approach; instead of trying to get the neutrons as well gathered in energy as possible, all energies are produced simultaneously. A high-energy proton beam hits a thick (in most cases stopping) target and lots of neutrons of all energies are produced, with typically a $1 / \mathrm{E}_{\mathrm{n}}$ spectrum. If the incident proton beam is bunched and the experiment target is placed at a rather large distance from the neutron production target, time-of-flight (TOF) methods can be used to determine the energy of the incident neutron on an event-by-event basis.

The advantage of such so called white beams is the total intensity, which is larger than for monoenergetic beams, but instead the intensity per energy interval is much lower at high energies. This can partly be compensated for by summing data over limited energy intervals, but still the intensity per such interval is lower. The advantage of being able to measure at many energies simultaneously is not worth much if you get insufficient statistics everywhere. As a consequence, white beams are restricted to experiments at low energies, where the intensities are large, or to 
high-energy reactions with rather large cross sections. Another feature is that white sources require event-by-event measurements. Experiments of effects with an energy dependence where the individual events cannot be distinguished cannot be performed at white beams. For experiments fulfilling the requirements above, white sources can, however, provide large quantities of very valuable information. This is especially true when excitation functions, i.e., the energy dependence of a cross section, is of particular interest.

\section{Nuclear data status}

It is a fairly limited class of reactions that are of interest for the further development of the applications under consideration. The most important are elastic scattering, inelastic neutron emission, light ion production, heavy ion production and fission.

Elastic scattering has been studied on a range of nuclei up to $96 \mathrm{MeV}$. At present, ten nuclei have been studied and results are either published or underway [7]. An overall uncertainty of about $5 \%$ has been achieved. A novel normalization method has been established that allows elastic scattering data to be normalized absolutely to about $3 \%$ uncertainty [8]. This method, however, works only for elastic scattering. Feasibility studies have shown that the technique as such works up to about $200 \mathrm{MeV}$, so these studies can be extended up in energy.

An experimental programme on inelastic neutron emission, i.e., ( $\mathrm{n}, \mathrm{xn}$ ') reactions is in progress [9]. Data have been taken on lead and iron, and the method as such seems to work. It is too early to quote a final uncertainty in the results, but $10 \%$ seems feasible.

Data on light ion production has been acquired on about ten nuclei at $96 \mathrm{MeV}$, and analysis is in progress $[10,11]$. At present, about half the data set has been published. Normalization has been obtained by simultaneous detection of $n p$ scattering at an angle where the cross section uncertainty can be estimated to about $5 \%$, which is the dominating uncertatinty in the final light ion production cross sections. These studies are presently being extended to $180 \mathrm{MeV}$.

Fission cross sections have been studied at many facilities up to about $200 \mathrm{MeV}$ energy. The energy dependencies of the cross sections agree fairly well in shape, but the absolute scale differs by up to $15 \%$. It is at present not clear what causes this. One possibility is the normalizations used. Another possible cause is that the sensitivity to low-energy neutrons is not under control for some of the experiments. Dedicated experiments to remedy this situation are underway.

In principle, fission cross sections can be measured up to several $\mathrm{GeV}$ using white beams with a very high initial proton energy, like at the CERN-nTOF facility [12]. The neutron beam intensity is very low, but the cross sections are large and it is possible to detect a major fraction of the fission fragments, resulting in reasonable statistical precision. A major problem, however, is normalization, since the beam intensity is very difficult to monitor at these very high energies.

There are only a few examples of other fission data than cross sections. This means that important fission parameters, like angular distributions, yields, etc., essentially remain to be investigated at high neutron energies.

\section{Possible future facilities}

As was discussed in the previous section, the prospects for development in the near future, i.e., 
within ten years, can be summarized to extension to about $200 \mathrm{MeV}$ of ongoing work on elastic scattering, inelastic neutron emission and light ion production at around $100 \mathrm{MeV}$, and fission studies of other parameters than the cross section.

If looking a bit further into the future, we can allow ourselves to be more visionary. To my opinion, the single most important problem to solve if we want a significant development of the field is normalization. At present, we inevitably end up with an uncertainty of about $5 \%$, because we have to normalize to a reference, typically $n p$ scattering, which is known to - at best - 5\%, and it is difficult to see how this can be radically improved upon in a short term with present techniques.

I consider energy resolution to be the second largest problem, with intensity on third place. These two are, however, to a large degree coupled. If you aim for good neutron-beam energy resolution, you have to pay by poor intensity and vice versa. It is presently close to inconceivable to produce neutrons at high energies with a resolution better than $1 \mathrm{MeV}$ with a reasonable intensity. The limited intensity puts severe constraints on the detection, in such a way that the detection often has to be performed with techniques that sacrifice resolution for efficiency, resulting in a final resolution of a few $\mathrm{MeV}$. This means that only in a few rare cases, final states can be resolved.

Recently, a way out of this dilemma has been proposed as a by-product of the CERN beta-beam facility [13] under consideration. The background is that neutrino physics has progressed rapidly the last few years, with the discovery of neutrino oscillations as the most visible example. Up to now, essentially all accelerator-produced neutrinos have been muon neutrinos, being the final product of pion decay. Electron neutrinos are much more difficult to produce in large amounts, because they require a nuclear beta decay for their creation.

At the proposed CERN beta-beam facility, production of suitable beta-emitting nuclei should be undertaken in an ISOLDE-like facility, and the produced nuclei should be post-accelerated to very high energy and stored in a decay ring of race-track shape. At these very high energies, hundreds of GeV/A, there is a very strong Lorentz boost, which means that the neutrino is emitted very close to the beam direction in the laboratory system, in spite of that the emission is isotropic in its moving reference frame. Thereby, intense neutrino beams can be produced. The idea is to build the decay ring so that one straight section points towards a distant neutrino detector to allow studies of electron neutrino oscillations.

Intense neutron beams could be a spin-off from that facility. It has been proposed to use two production targets, one for nuclei suited for neutrino emission in the decay ring, and one for betadelayed neutron emitters. Some neutron-rich nuclei beta decay to a nucleus that promptly emits a neutron, which typically has an energy of a few hundred $\mathrm{keV}$ in its rest frame. By accelerating the beta-delayed neutron emitters up to a few hundred $\mathrm{MeV}$ per nucleon, the Lorentz boost is sufficient to focus the beam to reasonable dimensions. All this can be done in parallel with the primary objective, since the accelerators for the neutrino emitters have a long cycle with a low duty factor.

The resulting neutron beam has an energy in the 100-500 MeV range with an energy resolution of about $1 \mathrm{MeV}$, and intensities of about $10^{11} \mathrm{n} / \mathrm{s}$ are estimated. This should be compared with $10^{6}$ for present-day technology, i.e., an improvement by a factor 100000 (!). With such intensities, only imagination sets the limit for what can be achieved.

If we now restrict the discussion to nuclear data for applications and turn to my problem list above, it seems feasible that we can address all of them through one experimental trick: tagging. If we use the neutron beam directly for experiments we have essentially only solved the intensity 


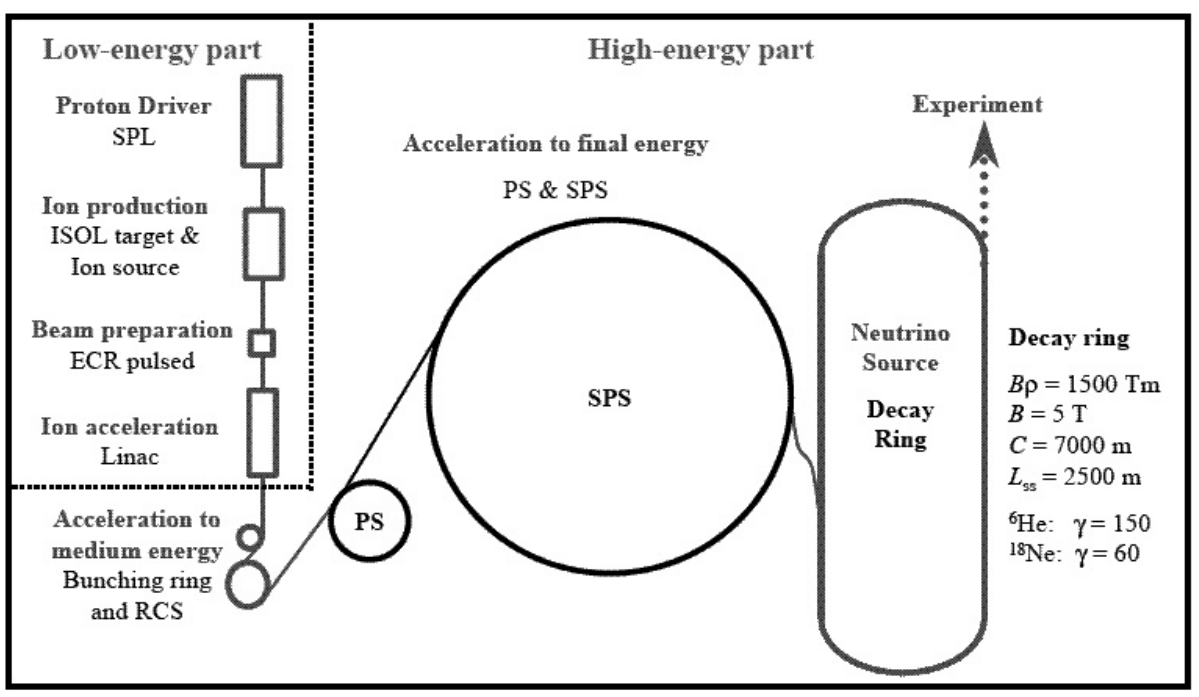

Figure 1: Overview of the proposed CERN beta-beam facility.

problem, but the other two remain; we end up in a $1 \mathrm{MeV}$ resolution due to the inherent energy spread, and we are still plagued by the normalization problem. Tagging means that we produce a secondary neutron beam of less intensity, but with much better known intensity. One candidate reaction is to let neutrons scatter from a hydrogen target, and the recoil proton is detected. Since this is a two-body final state, detection of the associated proton means that a neutron must have been scattered to the corresponding direction. Thereby, the normalization problem can be circumvented, since we count the neutrons one by one through the associated particle. If high-resolution tagging is performed, we can also know the neutron energy event by event far better than the initial neutron beam energy resolution. If the tagging is performed with a magnetic spectrometer, the tagger can be made rather insensitive to the ambient background, and a proton energy resolution of better than $100 \mathrm{keV}$ can be obtained, resulting in a comparable neutron energy resolution.

With reasonable estimates on tagger parameters, $10^{4}$ tagged neutrons with an energy resolution of $100 \mathrm{keV}$ should be possible to reach, given the beam intensity above. This might sound like a poor intensity, but with such a resolution, final states can be well resolved, which means that already a small number of events will result in a good precision. Moreover, since the intensity can be determined to about $1 \%$ in a typical tagger system, the accuracy is far better than what can be obtained today. In cases when the demands on energy resolution are not as stringent, a thicker tagger target can be used, resulting in increased intensity. This goes faster than linear, because with a worse resolution, the intensity at the tagger is increased, thicker secondary experimental targets can be used, and the detection limitations are less severe. Therefore, even with resolutions that are on the limit to be possible untagged today, we might have tagged beams of intensities exceeding what is presently available untagged in a not too distant future.

A second technique would be to use a similar production as above $(1-2 \mathrm{GeV}$ protons on a combined target-ion source) to produce ${ }^{6} \mathrm{He}$, which in turn would be accelerated to hit a target. Roughly, ${ }^{6} \mathrm{He}$ can be described as an $\alpha$ particle with two loosely attached neutrons. When hitting a 
target, the two neutrons are dissociated with a large probability, and continue along the direction of the incident beam with the incident velocity. The charged particles (the remaining ${ }^{6} \mathrm{He}$ and residual ${ }^{4} \mathrm{He}$ ) are bent by a magnet system and a clean neutron beam is produced. This latter technique does not have the potential to produce as intense fluxes as the beta-decay in flight, but on the other hand it requires much less advanced accelerators. This technique could possibly be installed at existing CERN facilities after some upgrades. Initial estimates indicate a factor a hundred to a thousand larger neutron fluxes than for present facilities to be within reach.

\section{Acknowledgments}

The information from Mats Lindroos is gratefully acknowledged. This work was financially supported by the Swedish Nuclear Fuel and Waste Management Company, the Swedish Nuclear Power Inspectorate, Ringhals AB, Forsmarks Kraftgrupp AB, the Swedish Defense Research Agency, the Swedish Nuclear Safety and Training Centre, and the European Union.

\section{References}

[1] A. Koning, et al., J. Nucl. Sci. Tech., Suppl. 2 (2002) 1161.

[2] J. Blomgren, in Proceedings of Workshop on Nuclear Data for Science \& Technology: Accelerator Driven Waste Incineration, Trieste, Italy, Sept. 10-21, 2001, eds. M. Herman, N. Paver, A. Stanculescu, ICTP lecture notes 12 (2002) 327.

[3] J. Blomgren, Nuclear data for accelerator-driven systems - Experiments above $20 \mathrm{MeV}$, in Proceedings of EU enlargement workshop on Neutron Measurements and Evaluations for Applications, Bucharest, Romania, October 20-23, 2004.

[4] J. Blomgren and N. Olsson, Radiat. Prot. Dosim. 103(4) (2003) 293.

[5] J. Blomgren, B. Granbom, T. Granlund, N. Olsson, Mat. Res. Soc. Bull. 28 (2003) 121.

[6] J. Blomgren, Nuclear Data for Single-Event Effects, in Proceedings of EU enlargement workshop on Neutron Measurements and Evaluations for Applications, Budapest, Hungary, November 5-8, 2003. EUR Report 21100 EN, Luxembourg: Office for Official Publications of the European Communities, ISBN 92-894-6041-5, European Communities, 2004.

[7] A. Hildebrand, et al., AIP Conference Proceedings 769 (2005) 853.

[8] J. Klug, et al., Phys. Rev. C 68, 064605 (2003).

[9] F.-R. Lecolley, private communication.

[10] V. Blideanu, et al., Phys. Rev. C 70 (2004) 014607.

[11] U. Tippawan, et al., Phys. Rev. C 69, 064609 (2004).

[12] L. Tassan-Got, private communication.

[13] The CERN beta-beam working group, http://cern.ch/beta-beam. 